\title{
The single degenerate channel for the progenitors of Type Ia supernovae
}

\author{
Xiangcun Meng ${ }^{1,2}$, Xuefei Chen ${ }^{1}$ and Zhanwen Han ${ }^{1}$ \\ ${ }^{1}$ National Astronomical Observatories/Yunnan Observatory, the Chinese Academy of Sciences, \\ Kunming, 650011, China, \\ email: conson859@msn.com \\ ${ }^{2}$ Graduate School of the Chinese Academy of Sciences
}

\begin{abstract}
We have carried out a detailed study of the single-degenerate channel for the progenitors of type Ia supernovae (SNe Ia). In the model, a carbon-oxygen white dwarf (CO WD) accretes hydrogen-rich material from an unevolved or a slightly evolved non-degenerate companion to increase its mass to Chandrasekhar mass limit. Incorporating the prescription of Hachisu et al. (1999a) for the accretion efficiency into Eggleton's stellar evolution code and assuming that the prescription is valid for all metallicities, we performed binary stellar evolution calculations for more than 25,000 close WD binary systems with various metallicities. The initial parameter spaces for $\mathrm{SNe}$ Ia are presented in an orbital period-secondary mass $\left(\log P_{\mathrm{i}}, M_{2}^{\mathrm{i}}\right)$ plane for each $Z$.

Adopting the results above, we studied the birth rate of SNe Ia for various $Z$ via binary population synthesis. From the study, we see that for a high $Z$, SNe Ia occur systemically earlier and the peak value of the birth rate is larger if a single starburst is assumed. The Galactic birth rate from the channel is lower than (but comparable to) that inferred from observations.

We also showed the distributions of the parameters of the binary systems at the moment of supernova explosion and the distributions of the properties of companions after supernova explosion. The former provides physics input to simulate the interaction between supernova ejecta and its companion, and the latter is helpful for searching the companions in supernova remnants.
\end{abstract}

Keywords. Binaries: close, stars: evolution, supernovae: general, white dwarf: metallicity

Although type Ia supernovae (SNe Ia) appear to be good cosmological distance indicators and have been applied successfully in determining cosmological parameters (e.g. $\Omega$ and $\Lambda$; Riess et al. 1998; Perlmutter et al. 1999), the exact nature of SNe Ia is still unclear, especially the progenitor model of SNe Ia (see the reviews by Hillebrandt \& Niemeyer 2000; Leibundgut 2000). At present, the single-degenerate Chandrasekhar model (Whelan \& Iben 1973; Nomoto, Thielemann \& Yokoi 1984) is the most widely accepted model. In the model, a CO WD accretes hydrogen-rich material from its companion until its mass reaches a mass of $\sim 1.378 M_{\odot}$ (close to Chandrasekhar mass, Nomoto, Thielemann \& Yokoi 1984), and then explodes as a SN Ia. The companion is probably a main sequence star or a slightly evolved star (WD+MS). The discovery of the potential companion of Tycho's supernova also verified the reliability of the WD + MS model (Ruiz-Lapuente et al. 2004; Ihara et al. 2007). The purpose of this paper is to study the progenitor model comprehensively and systematically.

\section{Binary evolution calculation}

We use the stellar evolution code of Eggleton $(1971,1972,1973)$ to calculate the binary evolutions of WD+MS systems. The code has been updated with the latest input physics 


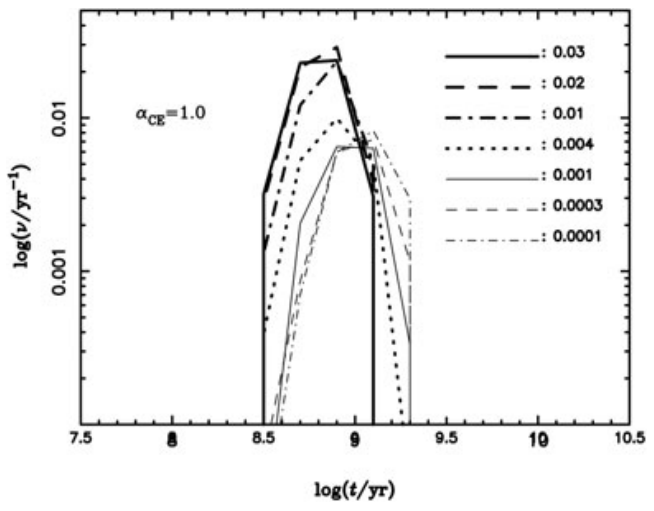

Figure 1. The evolution of the birth rate of SNe Ia for a single starburst of $10^{11} M_{\odot}$ for different metallicities with $\alpha_{\mathrm{CE}}=1.0$.

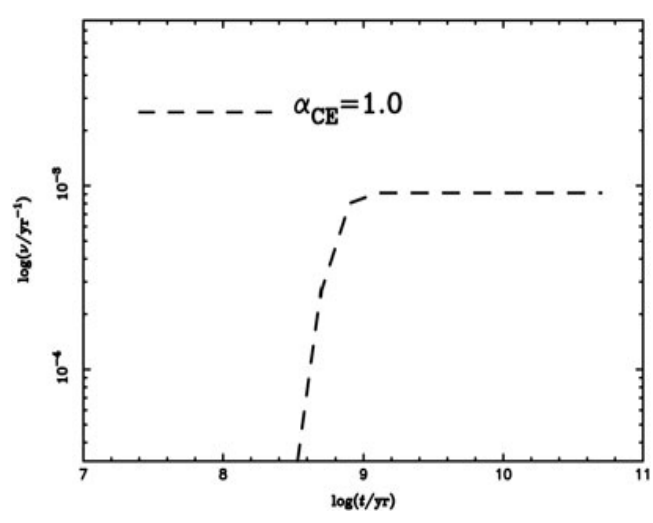

Figure 2. The evolution of the birth rate of $\mathrm{SNe}$ Ia for a constant star formation rate $\left(\mathrm{Z}=0.02, \mathrm{SFR}=5 M_{\odot} \mathrm{yr}^{-1}\right)$ with $\alpha_{\mathrm{CE}}=1.0$.

over the last three decades (Han, Podsiadlowski \& Eggleton 1994; Pols et al. 1995, Pols et al. 1998). Roche lobe overflow (RLOF) is treated within the code described by Han et al. (2000). Ten metallicities are chosen here (i.e. $Z=0.0001,0.0003,0.001,0.004,0.01$, $0.02,0.03,0.04,0.05$ and 0.06$)$. The opacity tables for these metallicties are compiled by Chen \& Tout (2007) from Iglesias \& Rogers (1996) and Alexander \& Ferguson (1994).

Instead of solving stellar structure equations of a WD, we adopt the prescription in Hachisu et al. (1999a) on the accretion of the hydrogen-rich material from its companion onto the WD based on an assumption of optically thick wind (Hachisu et al. 1996) (see Meng, Chen \& Han. 2008 for the prescription in details).

We calculated more than 25,000 WD+MS binary systems with various metallicities, and obtained a large, dense model grid. We summarize the final outcomes of all the binary evolution calculations in the initial orbital period-secondary mass $\left(\log P^{\mathrm{i}}, M_{2}^{\mathrm{i}}\right)$ planes (see Figs. 1 to 10 in Meng, Chen \& Han. 2008). The contours for SNe Ia are shown by solid lines in these figures. From these figures, we see that the contour moves from the left lower region to the right upper region with metallicity $\mathrm{Z}$ in the $\left(\log P^{\mathrm{i}}, M_{2}^{\mathrm{i}}\right)$ plane. This means that the progenitor systems for SNe Ia have a more massive companion and a longer orbital period for a high Z. We wrote our results into a FORTRAN code and the code can be downloaded on X. Meng's personal web site $h t t p: / / w w w . y n a o . a c . c n / \sim b p s /$ download /xiangcunmeng.htm.

\section{The results of binary population synthesis}

To investigate the birth rate of SNe Ia, we followed the evolution of $10^{7}$ binaries for various $Z$ with Hurley's rapid binary evolution code (Hurley et al. 2000, 2002). The results of grid calculations in section 1 are incorporated into the code. Since the code is valid just for $Z \leqslant 0.03$, only seven metallicities (i.e. $Z=0.03,0.02,0.01,0.004,0.001$, 0.0003 and 0.0001 ) are examined here. The primordial binary samples are generated in a Monte Carlo way and a circular orbit is assumed (see Meng, Chen \& Han. 2008 for details). The results are shown in Figs. 1 and 2. From Fig. 1, we see that most supernovae occur between 0.2 Gyr and 2 Gyr after star formation, and a high metallicity leads to a systematically earlier explosion time. The peak value of birth rate increases with metallicity $Z$. However, Fig 2 shows that the WD + MS channel can only account for about $1 / 3$ of the Galactic SNe Ia observed $\left(3-4 \times 10^{-3} \mathrm{yr}^{-1}\right.$, van den Bergh \& Tammann 1991; Cappellaro \& Turatto 1997). Therefore, there may be other channels or mechanisms contributing to SNe Ia. 


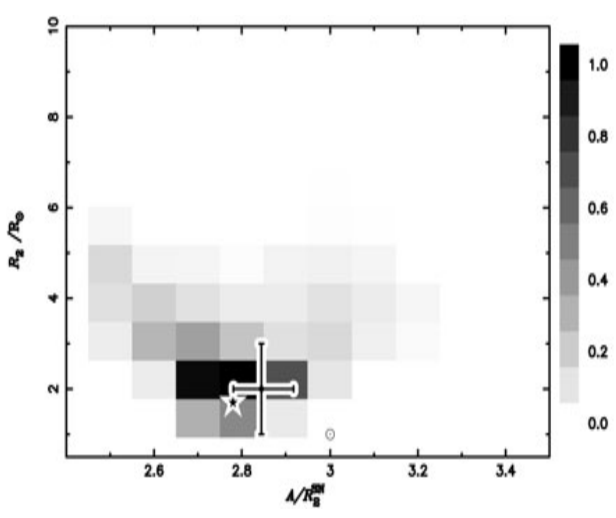

Figure 3. The distribution of the radii and the ratios of separations to radii of companions at the moment of supernova explosion for the case of $Z=0.02$ and $\alpha_{\mathrm{CE}}=1.0$. Cross represents the potential candidate of the companion of Tycho's supernova, Tycho G (RuizLapuente et al. 2004; Branch 2004). The length of the bars of the cross represents observational error. Solar symbol and pentacle represent the main-sequence and subgiant companion model in Marietta et al. (2000), respectively.

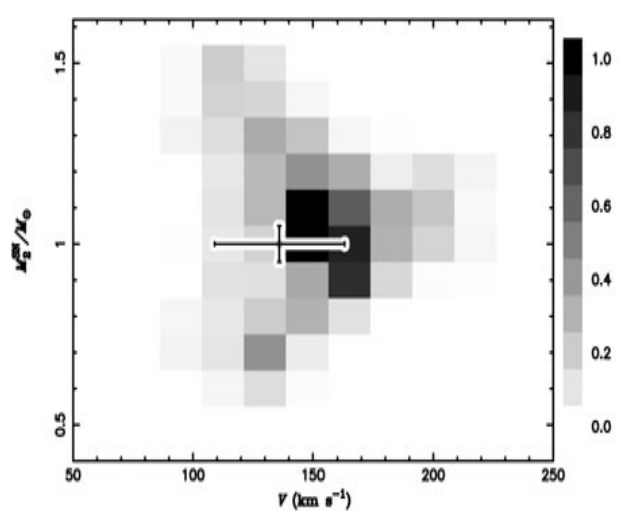

Figure 4. The distribution of the masses and the space velocities of companions in SN Ia remnants for $Z=0.02$ and $\alpha_{\mathrm{CE}}=1.0$. The cross represents the potential candidate of the companion of Tycho's supernova, Tycho G (Ruiz-Lapuente et al. 2004; Branch 2004), and the length of the bars of the cross represents observational errors.

We also showed the distributions of the parameters of the binary systems at the moment of supernova explosion (Fig. 3) and the properties of companions after supernova explosion (Fig. 4). The former may provide physics input when one simulates the interaction between supernova ejecta and its companion, and the latter may help to search for the companions in supernova remnants. From Fig. 4, we see that the properties of the potential companion of Tycho's supernova, Tycho G, are well consistent with our binary population synthesis results. We also noticed from Fig. 3 that the solar model used by Marietta et al. (2000) when they simulated the interaction between explosion ejecta and the companion is not a typical one (see the solar symbol in the figure).

A SN Ia remnant is not spherically symmetric due to the companion, and then the spectrum of the SN Ia may be polarized (Leonard \& Filippenko 2005). Based on the numerical simulation of Marietta et al. (2000) and Kasen et al. (2004), we found that at least $75 \%$ of all SNe Ia may be detected by spectropolarimetry. At present, almost all SNe Ia, which are observed by spectropolarimetry, have various degrees of polarization signal (Leonard et al. 2005).

Since there exists mass-loss from a progenitor binary system by an optically thick wind, the material lost from the system may become circumstellar materials (CSM). The CSM may be the origin of the color excess of SNe Ia. Based on the single degenerate model with optically thick wind and using a simple analytic method, we reproduced the distribution of the color excess of SNe Ia obtained from observation if a wind velocity of 10 $\mathrm{km} / \mathrm{s}$ is adopted. If the wind velocity is larger than $100 \mathrm{~km} / \mathrm{s}$, the reproduction is bad.

\section{Conclusion}

We systematically studied the single degenerate channel of SNe Ia, (i.e. WD+MS channel), and showed the parameter space leading to SNe Ia from this channel. Our work may provide help for the future studies of SNe Ia. We also provided the properties of 
companions after supernova explosion, which may help to search for the companion in SN Ia remnants. We found that at least $75 \%$ of all SNe Ia may be detected by spectropolarimetry, and then in the future, statistical studies about the spectropolarimetric observations of SNe Ia may verify the reliability of the WD + MS channel.

\section{Acknowledgements}

This work was in part supported by Natural Science Foundation of China under Grant Nos. 10433030, 10521001, 2007CB815406 and 10603013.

\section{References}

Alexander, D. R. \& Ferguson, J. W. 1994, ApJ 437, 879

Branch, D. 2004, Nature 431, 1044

Cappellaro, E. \& Turatto, M. 1997, in Ruiz-Lapuente P., Cannal R., Isern J., eds, Thermonuclear Supernovae. Kluwer, Dordrecht p. 77

Chen, X. \& Tout, C. A. 2007, ChJAA 7, 2, 245

Eggleton, P. P. 1971, MNRAS 151, 351

Eggleton, P. P. 1972, MNRAS 156, 361

Eggleton, P. P. 1973, MNRAS 163, 279

Hachisu, I., Kato, M., \& Nomoto, K. 1996, ApJ 470, L97

Hachisu, I., Kato, M., Nomoto, K., \& Umeda, H. 1999a, ApJ 519, 314

Han, Z., Podsiadlowski, P., \& Eggleton, P. P. 1994, MNRAS 270, 121

Han, Z., Tout, C. A., \& Eggleton, P. P. 2000, MNRAS 319, 215

Han, Z. \& Podsiadlowski, Ph. 2006, MNRAS 368, 1095

Hillebrandt, W. \& Niemeyer J. C. 2000, ARA\&A 38, 191

Hurley, J. R., Pols, O. R., \& Tout, C. A. 2000, MNRAS 315, 543

Hurley, J. R., Tout, C. A., \& Pols, O. R. 2002, MNRAS 329, 897

Iglesias, C. A. \& Rogers, F. J. 1996, ApJ 464, 943

Ihara, Y., Ozaki, J., Doi, M., et al. 2007, PASJ 59, 811, arXiv: 0706.3259

Kasen, D., Nugent, P., Thomas, R. C., \& Wang, L. 2004, ApJ 610, 876

Leibundgut, B. 2000, A\&ARv 10, 179

Leonard, D. C. \& Filippenko, A.V 2005, in Turatto M. et al., eds, in 1604-2004, Supernovae as Cosmological Lighthouses, (San Francisco: ASP), (astro-ph/0409518)

Leonard, D. C., et al. 2005, ApJ 632, 450

Marietta, E., Burrows, A., \& Fryxell, B. 2000, ApJS 128, 615

Meng, X., Chen, X., \& Han, X. 2008, arXiv: 0802.2471

Nomoto, K., Thielemann, F-K., \& Yokoi, K. 1984, ApJ 286, 644

Perlmutter, S., et al. 1999, ApJ 517, 565

Phillips, M. M. 1993, ApJ 413, L105

Pols, O. R., Tout, C. A., Eggleton, P. P., et al. 1995, MNRAS 274, 964

Pols, O. R., Schröder, K. P., Hurly, J. R., et al. 1998, MNRAS 298, 525

Reindl, B., Tammann, G. A., Sandage, A., et al. 2005, ApJ 624, 532

Riess, A. et al. 1998, AJ 116, 1009

Ruiz-Lapuente, P., et al. 2004, Nature 431, 1069

van den Bergh, S. \& Tammann, G. A. 1991, ARA\&A 29, 363

Whelan, J. \& Iben, I. 1973, ApJ 186, 1007

\section{Discussion}

J. Christensen-DalsgandD: Is the Tycho supernova the only Type Ia case where the companion may have been identified?

X.C. Meng: At present there is not others. Even for Tycho G, it is only a potential candidate of the companion of Tycho's supernova. 